\title{
Interlaboratory study of ethanol usage as an internal standard in direct determination of volatile compounds in alcoholic products
}

\author{
S. Charapitsa ${ }^{1}$, S. Sytova ${ }^{1}$, A. Korban ${ }^{1,2}$, L. Sobolenko ${ }^{1,2}$, V. Egorov ${ }^{2}$, S. Leschev ${ }^{2}$, M. Zakharov ${ }^{3}$, R. Čabala ${ }^{4}$, \\ R. Busarova ${ }^{5}$, I. Shestakovich ${ }^{6}$, A. Tolstouhova ${ }^{6}$, S. Ondroušek ${ }^{7}$, J. Vávra ${ }^{7}$, M. Yilmaztekin ${ }^{8}$, and T. Cabaroglu ${ }^{9}$ \\ ${ }^{1}$ Institute for Nuclear Problems of Belarusian State University, 220030, Bobruyskaya Str., 11, Minsk, Belarus \\ ${ }^{2}$ Chemistry Faculty, Department of Analytical Chemistry, Belarusian State University, Leningradskaya Str., 14, 220050, Minsk, Belarus \\ ${ }^{3}$ All-Russian Scientific Research Institute of the Brewing, Non-Alcoholic and Wine Industry - Branch of the V.M. Gorbatov Federal \\ Scientific Center of Food Systems of RAS, 119021, Rossolimo Str., 17, Moscow, Russia \\ ${ }^{4}$ Charles University, Faculty of Science, Department of Analytical Chemistry, Hlavova Str., 2030/8, 128 40, Prague 2, Czech Republic \\ 5 JS "Mosazervinzavod", 115088, Yuzhnoportovaya Str., 30, Moscow, Russia \\ ${ }^{6}$ Republican Centre for Hygiene, Epidemiology and Public Health, 220099, Kazintsa Str., 50, Minsk, Belarus \\ ${ }^{7}$ General Directorate of Customs, Customs Technical Laboratory, Budějovická Str., 7, 140 96, Prague 4, Czech Republic \\ ${ }^{8}$ Inonu University, Faculty of Engineering, Department of Food Engineering, 44280, Malatya, Turkey \\ ${ }^{9}$ Cukurova University, Faculty of Agriculture, Department of Food Engineering, 01330, Adana, Turkey
}

\begin{abstract}
A collaborative interlaboratory study on the method of direct quantitation of volatile compounds in spirit drinks and alcoholic products was conducted. The discussed method applies ethanol, the major volatile component of an alcoholic product, as an internal standard. In this study 9 laboratories from 4 different countries were supplied with standard solutions for gas chromatographic measurements. Five aqueous ethanol $40 \%(\mathrm{v} / \mathrm{v})$ standard solutions containing target compounds in concentrations ranging from $10 \mathrm{mg} / \mathrm{L}$ to $400 \mathrm{mg} / \mathrm{L}$ of absolute alcohol were prepared and sent to the participants for quantification of acetaldehyde, methyl acetate, ethyl acetate, methanol, 2-propanol, 1-propanol, 2-methyl-1-propanol, 1-butanol and 3-methyl-1-butanol. The interlaboratory study was evaluated according to the ISO 5725 standards and the Eurachem guide. The within-laboratory precision varied between $0.4 \%$ and $7.5 \%$ for all samples and compounds, showing a sufficiently high repeatability of the method. The between-laboratory precision was found to vary within a satisfactory range of $0.5 \% \div 10.0 \%$. Precision of the method was well within the range predicted by the Horwitz equation for all analytes. The analysis of trueness showed that the bias of the method is insignificant at the significance level $\alpha=5 \%$. The determined concentrations of the analytes compared well to the gravimetrical values thus showing very satisfactory accuracy of the method. The results of the interlaboratory study confirmed that "Ethanol as Internal Standard" method is robust and reliable and can be used as a standard reference method for analysing volatile compounds in water-ethanol samples. The possibilities of method validation according to the previously obtained experimental data were shown.
\end{abstract}

\section{Introduction}

This paper is a logical continuation of a single-laboratory validation of the "Ethanol as Internal Standard" method [1]. The latter was already proved to be accurate and precise during single-laboratory experiment [2] and interlaboratory approbation within 3 laboratories [3]. The discussed method is applied for gas chromatographic (GC) direct quantification of volatile compounds in spirit drinks and other ethanol containing samples. The theoretical background and application algorithm of the method are very close to those described in the European regulation [4]. It should be noted that method of Internal Standard (IS) is used worldwide for the quantification of volatile compounds in alcoholic products [4-8]. The major difference between cited methods and the proposed one consists in employing ethanol, the main volatile organic component, as an IS. This uncommon approach allows to skip the necessity of IS compound addition into both calibration and test samples and the necessity of sample density and strength establishment. The term "strength" is defined as ethanol volumetric content in the sample. Further we will use a more common ABV (alcohol by volume) abbreviation during the description of analysed samples. Eventually, the absence of these manual procedures leads to the fact that "Ethanol as IS" method is simpler, cheaper, more rapid and robust in comparison with currently used methods. These benefits originate from the fact that according to the abovementioned regulatory documents volatile compounds concentrations must be finally expressed in mass per litre of absolute alcohol units $(\mathrm{mg} / \mathrm{L} \mathrm{AA}, \mathrm{g} / \mathrm{L}$ AA etc.). That is why the approach of employing ethanol as an internal standard is a logical step towards improvement of quality control analysis 
Table 1. Concentrations of volatile components in the prepared standard solutions and their uncertainties.

\begin{tabular}{|l|c|c|c|c|c|}
\hline \multirow{2}{*}{ Compound } & \multicolumn{5}{|c|}{ Concentration, mg/L AA } \\
\cline { 2 - 6 } & SS-2 & SS-1 & SS-D & SS-C & SS-B \\
\hline acetaldehyde & $10.7 \pm 0.2$ & $47.8 \pm 1.0$ & $183 \pm 4$ & $227 \pm 5$ & $414 \pm 6$ \\
\hline methyl acetate & $8.7 \pm 0.2$ & $45.4 \pm 0.9$ & $182 \pm 4$ & $230 \pm 5$ & $427 \pm 6$ \\
\hline ethyl acetate & $9.4 \pm 0.2$ & $47.8 \pm 1.0$ & $189 \pm 4$ & $232 \pm 5$ & $423 \pm 6$ \\
\hline methanol & $22.6 \pm 0.5$ & $59.8 \pm 1.2$ & $198 \pm 4$ & $241 \pm 5$ & $424 \pm 6$ \\
\hline 2-propanol & $10.1 \pm 0.2$ & $46.3 \pm 0.9$ & $180 \pm 4$ & $225 \pm 4$ & $405 \pm 6$ \\
\hline ethanol & \multicolumn{5}{|c|}{789,300} \\
\hline 1-propanol & $9.7 \pm 0.2$ & $47.8 \pm 1.0$ & $186 \pm 4$ & $231 \pm 5$ & $418 \pm 6$ \\
\hline isobutanol & $9.7 \pm 0.2$ & $48.8 \pm 1.0$ & $190 \pm 4$ & $238 \pm 5$ & $431 \pm 6$ \\
\hline 1-butanol & $9.2 \pm 0.2$ & $47.2 \pm 0.9$ & $185 \pm 4$ & $231 \pm 5$ & $417 \pm 6$ \\
\hline isoamylol & $9.3 \pm 0.2$ & $46.9 \pm 1.0$ & $183 \pm 4$ & $228 \pm 5$ & $414 \pm 6$ \\
\hline
\end{tabular}

of alcoholic products. Current work was undertaken in analogy with interlaboratory studies conducted 20 years ago [9].

\section{Materials and methods}

\subsection{Reagents}

High-purity ethanol with $96 \%$ ABV was supplied by Dyatlovo Distillery Plant "Algon" (Belarus). Pure deionized water was purchased at JSC "Integral" (Belarus). The following chemical standards were purchased from Sigma Aldrich (Alcobendas, Madrid, Spain) with the highest purity available (more than 99\%): acetaldehyde, methyl acetate, ethyl acetate, methanol, 2propanol, 1-propanol, 2-methyl-1-propanol (isobutanol), 1-butanol, 3-methyl-1butanol (isoamylol).

\subsection{Preparation of standard solutions}

Hydroalcoholic solution (HS) with 40\% ABV was prepared from ethanol and deionized water. Five standard solutions (SS): SS-B, SS-C, SS-D, SS-1 and SS2 were gravimetrically prepared in the HS matrix. To prepare the most saturated SS-B the following volatile compounds were added to the HS: acetaldehyde, methyl acetate, ethyl acetate, methanol, 2-propanol, 1-propanol, isobutanol, 1-butanol, isoamylol. Samples SS-C, SS-D, SS-1 and SS-2 were prepared by dilution of SS-B or SS-C with HS. The concentrations of volatile compounds with the corresponding uncertainties in the prepared standard solutions are shown in Table 1.

\subsection{Storage and transportation}

Prepared samples were put into standard $1.5 \mathrm{ml}$ microvials with crimp caps and sent to the participants by regular mail in packages, protecting samples from sunlight and mechanical damage. Each microvial was marked with a QR code for digital identification.

\subsection{Analysis}

Analysis was performed with gas chromatography with flame ionization detection. Laboratories were permitted to select gas chromatographic system and conditions allowing acceptable separation of the compounds as determined in items $6.3-6.4$ of European regulation [4]. Each laboratory was required to measure each SS in

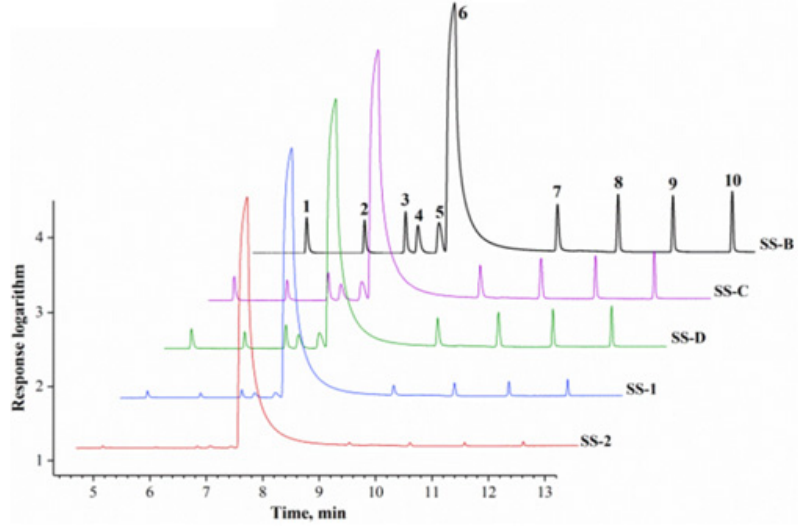

Figure 1. Chromatograms of the prepared standard solutions. 1 - acetaldehyde; 2 - methyl acetate; 3 - ethyl acetate;

4 - methanol; 5 - 2-propanol; 6 - ethanol; 7 - 1-propanol;

8 - isobutanol; 9 - 1-butanol; 10 - isoamylol.

triplicate under repeatability conditions. The example of obtained chromatograms of five prepared SS in logarithmic scale is shown in Fig. 1.

\section{Calculations}

\subsection{Determination of concentrations of volatile compounds in the prepared solutions}

Although the preparation of the samples was done in a gravimetrical way it was very important to take into consideration concentrations of volatile compounds in the initial HS used as a matrix.

Concentrations of volatile compounds in SS-B in $\mathrm{mg} / \mathrm{L}$ $\mathrm{AA}$ and $\mathrm{mg} / \mathrm{mg}$ units were calculated according to the Eqs. $(1,2)$ :

$$
\begin{gathered}
C_{i}^{B}(m g / L A A)=\rho_{E t h} \cdot \frac{C_{i}^{i} \cdot m_{i}^{B}+C_{i}^{H S} \cdot m_{H S}^{B}}{C_{E t h}^{H S} \cdot m_{H S}^{B}}, \\
C_{i}^{B}(\mathrm{mg} / \mathrm{mg})=\frac{C_{i}^{i} \cdot m_{i}^{B}+C_{i}^{H S} \cdot m_{H S}^{B}}{M_{B}},
\end{gathered}
$$

where $\rho_{E t h}$ - ethanol density, 789,300 mg/L; $C_{i}^{i}-$ concentration of the $i$-th volatile compound in the $i$-th chemical standard, $\mathrm{mg} / \mathrm{mg} ; m_{i}^{B}$ - mass of the $i$-th volatile compound added to the HS for the SS-B preparation, mg; $C_{i}^{H S}$ concentration of the $i$-th volatile compound in HS, $\mathrm{mg} / \mathrm{mg}$; $C_{E t h}^{H S}$ - concentration of ethanol in $\mathrm{HS}, \mathrm{mg} / \mathrm{mg} ; m_{H S}^{B}-$ mass of the HS added for the SS-B preparation, mg; $M_{B}-$ final mass of the prepared SS-B, mg.

Concentrations of volatile components in the SS-C, SSD, SS-1 and SS-2 in mg/L AA and $\mathrm{mg} / \mathrm{mg}$ units were calculated according to the Eqs. $(3,4)$ :

$$
C_{i}^{C, . ., 2}(m g / L A A)=\rho_{E t h} \cdot \frac{C_{i}^{B} \cdot m_{B}^{C, . ., 2}+C_{i}^{H S} \cdot m_{H S}^{C, \ldots, 2}}{C_{E t h}^{B} \cdot m_{B}^{C, \ldots, 2}+C_{E t h}^{H S} \cdot m_{H S}^{C, \ldots, 2}},
$$

$$
C_{i}^{C, \ldots, 2}(m g / m g)=\frac{C_{i}^{B} \cdot m_{B}^{C, ., 2}+C_{i}^{H S} \cdot m_{H S}^{C, \ldots, 2}}{M_{C, \ldots, 2}},
$$

where $C_{i}^{B}$ - concentration of the $i$-th volatile compound in the SS-B, $\mathrm{mg} / \mathrm{mg} ; m_{B}^{C, . ., 2}$ - mass of the SS-B added for 
the SS-C, SS-D, SS-1 or SS-2 preparation, $\mathrm{mg} ; m_{H S}^{C, . ., 2}-$ mass of the HS added for the SS-C, SS-D, SS-1 or SS2 preparation, mg; $C_{E t h}^{B}-$ ethanol concentration in SS-B, $\mathrm{mg} / \mathrm{mg} ; M_{C, \ldots, 2}-$ final mass of the prepared SS-C, SS-D, SS-1 or SS-2, mg.

Concentrations of volatile compounds in HS were calculated using the method of successive iterations. Firstly, the SS-C was measured in triplicate. Then a singlepoint calibration was done, it consisted in calculation of zero-order approximation Relative Response Factors $R R F_{i}^{E t h}(0)$ relatively to ethanol according to the following formula:

$$
R R F_{i}^{E t h}(0)=\frac{C_{i}^{s t}(0)}{A_{i}^{s t}} \cdot \frac{A_{E t h}^{s t}}{\rho_{E t h}},
$$

where $C_{i}^{s t}(0)$ is a concentration of the $i$-th volatile compound in SS-C, excluding it's concentrations in HS itself, mg/L AA units; $A_{i}^{s t}$ and $A_{E t h}^{s t}$ are detector responses for the $i$-th volatile compound and ethanol in the SS-C, correspondingly, a.u.

Then the HS was measured and zero-order approximation concentrations of volatile compounds were calculated according to the formula (6):

$$
C_{i}^{H S}(0)=R R F_{i}^{E t h}(0) \cdot \frac{A_{i}^{H S}}{A_{E t h}^{H S}} \cdot \rho_{E t h},
$$

where $\mathrm{A}_{\mathrm{i}}^{\mathrm{HS}}$ and $\mathrm{A}_{\mathrm{Eth}}^{\mathrm{HS}}$ - detector responses for the $i$-th volatile compound and ethanol in the HS, correspondingly, a.u. The conversion of concentration of the $i$-th volatile compound from $\mathrm{mg} / \mathrm{L} \mathrm{AA}$ to $\mathrm{mg} / \mathrm{mg}$ in $\mathrm{HS}$ was done according to the Eq. (7):

$$
C_{i}^{H S}(0, m g / m g)=\frac{C_{i}^{H S}(0, m g / L A A) \cdot A B V(\%)}{\rho_{H S} \cdot 100 \%},
$$

where $\rho_{\mathrm{HS}}-\mathrm{HS}$ density.

After that, values of $R R F_{i}^{E t h}$ for each $i$-th volatile compound were recalculated, using the clarified values of concentrations of the $i$-th volatile compound in SS-C according to the Eq. (3). Recalculations were repeated until the difference between the concentrations was less than $10^{-10} \mathrm{mg} / \mathrm{mg}$.

\subsection{Calibration and analysis}

A single-point calibration was done by usage of the SSC. Values of relative response factors $R R F_{i}^{E t h}$ for each $i$ th volatile compound relatively ethanol were determined according to the following formula:

$$
R R F_{i}^{E t h}=\frac{C_{i}^{s t}}{A_{i}^{s t}} \cdot \frac{A_{E t h}^{s t}}{\rho_{E t h}},
$$

where all values have the same meaning as in Eq. (5), except of $C_{i}^{s t}$, which represented final $i$-th concentration in $\mathrm{mg} / \mathrm{L} \mathrm{AA}$ after the iteration method, described in i. 3.1.

Concentration of the $i$-th volatile compound in $\mathrm{mg} / \mathrm{L}$ AA dimension was determined according to the following equation:

$$
C_{i}=R R F_{i}^{E t h} \cdot \frac{A_{i}}{A_{E t h}} \cdot \rho_{E t h},
$$

where all values have the same meaning as in Eq. (6), except $R R F_{i}^{E t h}$, which represented final relative response factor after the iteration method, described in i. 3.1.

\subsection{Metrological evaluation}

The statistical analysis of the obtained results, namely the detection and elimination of outliers, the precision parameters evaluation (repeatability, reproducibility and trueness), was based on the number of ISO 5725:1994 standards $[10,11]$.

\subsubsection{Outliers}

Cochran's and Grubbs' tests were performed to detect and eliminate outliers. Firstly, an upper-tail Cochran test was performed for the comparison of the interlaboratory variances. Secondly, a two-tailed single Grubbs' and a paired Grubbs' tests were applied according to the ISO 5725:1994 [10,11]. Both these methods allowed us to check for outliers within and among the participant laboratories.

\subsubsection{Z-scores}

Laboratory's performance was assessed by means of $z$-scores [12]. This parameter was determined for each result reported by the laboratories according to the following formula:

$$
z=\frac{\bar{x}-X}{s}
$$

where $\bar{x}$ - participant result; $X$ - assigned preparation value; $s$ - standard deviation based on participant's results. Calculated z-scores were evaluated according to the ISO/IEC 17043:2010 [13]. The values of $|z| \leq 2.0$ were assessed as acceptable, $2.0 \leq|\mathrm{z}| \leq 3.0$ - questionable and $|z| \geq 3.0$ - unacceptable.

\subsubsection{Linearity}

Graphs of linearity of the method for different laboratories were drawn with OriginPro 9.5 software (OriginLab Corporation, Northampton, MA, USA). The coefficients of determination $\mathrm{R}^{2}$ were also obtained by OriginPro 9.5 .

\subsubsection{Limit of Quantification (LOQ)}

LOQs of each individual volatile compound were estimated for each laboratory according to the [14] by usage SS-2 as the solution with lowest concentrations of impurities:

$$
L O Q=k \cdot \frac{s_{0}}{\sqrt{n}}
$$

where $s_{0}$ - standard deviation from measurement SS-2; $n$ number of replicate observations, $k=10$.

\subsubsection{Accuracy and precision}

For accuracy and precision estimation the three following variances: $S_{L}^{2}$ (interlaboratory), $S_{r}^{2}$ (repeatability) and $S_{R}^{2}$ (reproducibility) were calculated according to the ISO 5725:1994 [10,11]. The limits of repeatability $r$ and reproducibility $R$ were calculated according to the $[10,11]$ and [14]. 
Table 2. The obtained calibration coefficients relatively to ethanol.

\begin{tabular}{|l|c|c|c|c|c|c|c|c|c|}
\hline \multirow{2}{*}{ Compound } & \multicolumn{10}{|c|}{ Participant } \\
\cline { 2 - 11 } & 1 & 2 & 3 & 4 & 5 & 6 & 7 & 8 & 9 \\
\hline acetaldehyde & 1.674 & 2.157 & 1.162 & 1.313 & 1.275 & 1.425 & 1.551 & 1.185 & 1.512 \\
\hline methyl acetate & 1.678 & 2.408 & 1.307 & 1.727 & 1.652 & 1.611 & 1.790 & 1.413 & 1.746 \\
\hline ethyl acetate & 1.201 & 1.842 & 0.955 & 1.125 & 1.106 & 1.121 & 1.275 & 1.000 & 0.996 \\
\hline methanol & 1.304 & 1.872 & 1.182 & 1.291 & 1.290 & 1.298 & 1.464 & 1.173 & 1.335 \\
\hline 2-propanol & 0.885 & 1.282 & 0.756 & 0.880 & 0.895 & 0.896 & 0.992 & 0.843 & N/A \\
\hline ethanol & \multicolumn{10}{|c|}{1.000} \\
\hline 1-propanol & 0.729 & 1.015 & 0.645 & 0.729 & 0.736 & 0.753 & 0.800 & 0.665 & 0.757 \\
\hline isobutanol & 0.615 & 0.887 & 0.537 & 0.597 & 0.596 & 0.640 & 0.687 & 0.561 & 0.639 \\
\hline 1-butanol & 0.652 & 0.897 & 0.585 & 0.654 & 0.653 & 0.672 & 0.708 & 0.591 & 0.683 \\
\hline isoamylol & 0.598 & 0.819 & 0.531 & 0.587 & 0.586 & 0.613 & 0.649 & 0.547 & 0.628 \\
\hline
\end{tabular}

\subsubsection{Horwitz}

The predicted standard deviation of reproducibility $R S D_{R(H)} \quad$ (\%) was calculated with the Horwitz equation [15]:

$$
R S D_{R(H)}=2^{(1-0.5 \cdot \log C)},
$$

where $C$ - measured analyte concentration expressed as a decimal (e.g., $1 \mathrm{mg} / 100 \mathrm{mg}=0.01)$. The transfer of the directly obtained concentrations in $\mathrm{mg} / \mathrm{L}$ AA units into $\mathrm{mg} / \mathrm{kg}$ units was done in analogy with Eq. (7).

The predicted standard deviation of repeatability $R S D_{r(H)}(\%)$ was calculated according to the following formula:

$$
R S D_{r(H)}=0.66 \cdot R S D_{R(H)} .
$$

\subsubsection{Horrat}

The Horrat values [16] were calculated in order to compare actual measured precision with the precision predicted using the Eqs. $(12,13)$ according to the following formulas:

$$
\begin{aligned}
\text { Horrat }_{r} & =\frac{R S D_{r}}{R S D_{r(H)}}, \\
\text { Horrat }_{R} & =\frac{R S D_{R}}{R S D_{R(H)}} .
\end{aligned}
$$

The evaluation of obtained values was done in a traditionally accepted way. Horrat value of 1 indicated satisfactory interlaboratory precision, whereas a value of $>2$ indicated unsatisfactory precision.

\subsubsection{Trueness}

For the estimation of trueness of the method bias $\delta$, standard deviation of bias $S_{\delta}$ and confidence interval for the bias were calculated in accordance with ISO 5725:1994 $[10,11]$. It's necessary to point out that according to our knowledge there were no interlaboratory experiments including the calculation of biases while quantitation of volatile compounds in spirit drinks.

\subsubsection{Uncertainty}

The standard uncertainty, $u$, of the results was calculated according to the Eurolab [17] and Eurachem [18] guidelines using the following formula (10):

$$
u=\sqrt{S_{R}^{2}+S_{\delta}^{2}+u_{r e f}^{2}+\delta^{2}},
$$

where $u_{r e f} i$ s the uncertainty of the assigned value, calculated according to the Eurolab [17], using Eqs. (S.1-S.4). The expanded uncertainty $U$ was calculated as doubled $u$.

\section{Results and discussion}

The obtained calibration coefficients are presented in Table 2.

It was found that calibration coefficients obtained at similar instruments are very close to each other, which was already discussed [19]. The authors are sure that this fact raises the possibility of RRF coefficients tabulation among similar instruments making the calibration procedure simpler and more sustainable. In any case, the scarce amount of currently available data raises the need for further research prior to coming to this conclusion.

First, all obtained results were checked for the presence of outliers. Figures S.1-S.4 and Tables S.1-S.4 contain the information about calculated $\mathrm{z}$-scores and revealed outliers which were not taken into further statistical calculations. Here and further all statistical data doesn't include SS-C as this sample was employed as a calibrator.

The linearity of the method was evaluated by creation of linear dependences of $i$-th to ethanol relative detector responses on $i$-th to ethanol relative $\mathrm{mg} / \mathrm{L} \mathrm{AA}$ concentrations. The obtained determination coefficients $\mathrm{R}^{2}$ are presented in Table 3; the obtained linear graphs are presented in Figures S.5-S.11. The obtained results fully correlate with our previous experiments [1-3] and prove high linearity of the tested method.

The calculated LOQs are shown in Table 4. The obtained results prove that the method is applicable for measuring lowest required volatiles amounts [20], for instance in vodka and pure ethyl alcohol. The results of the rest of discussed metrological tests are presented in Tables S.5-S.8.

\subsection{The possibilities of method validation}

In accordance with the requirements of ISO/IEC 17043: 2010 [13], a method must be validated before an application in a routine laboratory practice. Here we perform possible algorithms of single and interlaboratory validation of the method.

\subsubsection{Single laboratory validation}

The discussed method can be easily validated within one laboratory on the basis of the data which was previously 
Table 3. The obtained determination coefficients $R^{2}$.

\begin{tabular}{|c|c|c|c|c|c|c|c|c|c|}
\hline \multirow{2}{*}{ Compound } & \multicolumn{9}{|c|}{ Participant } \\
\cline { 2 - 11 } & 1 & 2 & 3 & 4 & 5 & 6 & 7 & 8 & 9 \\
\hline acetaldehyde & 0.9999 & 0.9992 & 0.9999 & 0.9999 & 0.9962 & 0.9988 & 0.9999 & 0.9993 & 0.9999 \\
\hline methyl acetate & 0.9999 & 0.9994 & 0.9999 & 0.9999 & 0.9968 & 0.9989 & 0.9999 & 0.9993 & 0.9999 \\
\hline ethyl acetate & 0.9999 & 0.9994 & 0.9999 & 0.9999 & 0.9963 & 0.9988 & 0.9999 & 0.9992 & 0.9999 \\
\hline methanol & 0.9999 & 0.9999 & 0.9999 & 0.9999 & 0.9956 & 0.9988 & 0.9999 & 0.9991 & 0.9999 \\
\hline 2-propanol & 0.9999 & 0.9999 & 0.9999 & 0.9999 & 0.9963 & 0.9988 & 0.9999 & 0.9993 & N/A \\
\hline 1-propanol & 0.9999 & 0.9993 & 0.9999 & 0.9999 & 0.9962 & 0.9989 & 0.9999 & 0.9993 & 0.9999 \\
\hline isobutanol & 0.9999 & 0.9994 & 0.9999 & 0.9999 & 0.9962 & 0.9988 & 0.9999 & 0.9993 & 0.9999 \\
\hline 1-butanol & 0.9999 & 0.9994 & 0.9999 & 0.9999 & 0.9961 & 0.9989 & 0.9999 & 0.9993 & 0.9999 \\
\hline isoamylol & 0.9999 & 0.9994 & 0.9999 & 0.9999 & 0.9961 & 0.9989 & 0.9999 & 0.9993 & 0.9999 \\
\hline
\end{tabular}

Table 4. The limits of quantification in $\mathrm{mg} / \mathrm{L}$ AA units.

\begin{tabular}{|l|c|c|c|c|c|c|c|c|c|}
\hline \multirow{2}{*}{ Compound } & \multicolumn{10}{|c|}{ Participant } \\
\cline { 2 - 12 } & 1 & 2 & 3 & 4 & 5 & 6 & 7 & 8 & 9 \\
\hline acetaldehyde & 1.0 & 1.2 & 0.6 & 3.8 & 0.4 & 1.1 & 0.4 & 1.1 & 1.8 \\
\hline methyl acetate & 0.7 & 0.8 & 0.5 & 0.8 & 1.8 & 0.5 & 1.0 & 0.5 & 1.8 \\
\hline ethyl acetate & 0.2 & 1.8 & 0.8 & 0.7 & 0.5 & 1.1 & 0.4 & 1.1 & 3.3 \\
\hline methanol & 0.7 & 3.7 & 0.8 & 2.9 & 2.4 & 1.8 & 0.2 & 1.8 & 7.1 \\
\hline 2-propanol & 0.6 & 11.0 & 0.3 & 0.9 & 0.8 & 1.5 & 0.2 & 1.5 & N/A \\
\hline 1-propanol & 0.4 & 0.3 & 0.5 & 1.7 & 0.3 & 2.8 & 1.6 & 2.8 & 1.0 \\
\hline isobutanol & 0.4 & 0.9 & 0.3 & 0.8 & 0.5 & 0.4 & 0.9 & 0.4 & 2.0 \\
\hline 1-butanol & 0.9 & 1.8 & 0.1 & 0.5 & 0.7 & 0.6 & 1.1 & 2.8 & 1.0 \\
\hline isoamylol & 0.2 & 1.8 & 0.7 & 1.0 & 0.8 & 1.8 & 0.2 & 1.8 & 1.6 \\
\hline
\end{tabular}

Table 5. Volatile compounds concentrations, average peak area and RRFs for both traditional and advance IS methods.

\begin{tabular}{|c|c|c|c|c|c|}
\hline \multirow{2}{*}{ Compound } & \multicolumn{2}{|c|}{ Concentration } & \multirow{2}{*}{ Average peak area, a.u. } & \multicolumn{2}{|c|}{ Average calibration coefficient } \\
\hline & $\mathrm{mg} / \mathrm{L}$ & $\mathrm{mg} / \mathrm{L} \mathrm{AA}$ & & $R R F_{i}^{E t h}$ & $R R F_{i}^{3-p e n t a n o l}$ \\
\hline acetaldehyde & 200 & 501 & 9.489 & 1.319 & 2.011 \\
\hline methyl acetate & 261 & 652 & 9.406 & 1.733 & 2.642 \\
\hline ethyl acetate & 264 & 660 & 12.940 & 1.275 & 1.945 \\
\hline methanol & 231 & 577 & 10.122 & 1.427 & 2.175 \\
\hline ethanol & 315,720 & 789,300 & 19.744 & 1.000 & 1.525 \\
\hline 2-butanol & 237 & 593 & 19.097 & 0.777 & 1.184 \\
\hline 1-propanol & 231 & 576 & 18.108 & 0.796 & 1.214 \\
\hline isobutanol & 238 & 595 & 22.031 & 0.676 & 1.030 \\
\hline 3-pentanol (Traditional IS) & 233 & 583 & 22.234 & 0.656 & 1.000 \\
\hline 1-butanol & 240 & 600 & 21.042 & 0.713 & 1.087 \\
\hline 2-methyl-1-butanol & 234 & 585 & 22.322 & 0.656 & 1.000 \\
\hline 3-methyl-1-butanol & 232 & 581 & 22.590 & 0.643 & 0.980 \\
\hline
\end{tabular}

obtained during the validation of traditional IS method. No other measurements or manual procedures except those indicated in standards [4-8] are required.

Here we perform a detailed algorithm of method validation in one laboratory through the example of earlier undertaken validation [21]. The experimental part was done in accordance with EC 2870/2000 [4]. For the first step, namely calibration, we recalculated volatiles concentrations in calibration solution into $\mathrm{mg} / \mathrm{L}$ AA units. There were no difficulties as exact amounts of added ethanol and volatile compounds in the prepared standard solutions were known. After that we established the ethanol peak area in the chromatograms of the calibration solution "C" and calculated $R R F_{i}^{E t h}$ for all volatile components relatively to ethanol according to the Eq. (8). Table 5 contains concentrations of analysed volatile compounds in calibration solution in two dimensions, average established peak area and calibration coefficient values for both traditional and suggested IS methods.

Then the concentrations of volatile compounds in standard solutions " 0.05 "-"2.0" were recalculated into $\mathrm{mg} / \mathrm{L}$ AA units with "Ethanol as IS" method. These solutions were prepared to check the linearity properties of the traditional IS method. We employed them for the same goal but for the suggested method. By analogy with chromatograms of the calibration solution we only determined ethanol peak area and recalculated concentrations in these standard solutions according to the Eq. (9). Then the linearity plots were created and compared with those of the traditional IS method. Thus, determination coefficients $R^{2}$ were not lower than 0.9981 and 0.9999 for the traditional and novel IS methods, correspondingly.

The RSDs of the concentrations of these solutions were then evaluated and compared. This data can be 
Table 6. RSD of the concentrations for both traditional and novel IS methods.

\begin{tabular}{|c|c|c|c|c|c|c|c|c|}
\hline \multirow{3}{*}{ Compound } & \multicolumn{8}{|c|}{ RSD, \% } \\
\hline & \multicolumn{2}{|c|}{ "0.05" } & \multicolumn{2}{|c|}{ “0.1" } & \multicolumn{2}{|c|}{$" 0.5 "$} & \multicolumn{2}{|c|}{ "2.0" } \\
\hline & Novel & Trad & Novel & Trad & Novel & Trad & Novel & Trad \\
\hline acetaldehyde & 4.3 & 4.1 & 1.8 & 2.0 & 0.4 & 0.2 & 0.9 & 1.0 \\
\hline methyl acetate & 2.6 & 2.9 & 2.1 & 2.2 & 0.3 & 0.2 & 1.4 & 1.5 \\
\hline ethyl acetate & 3.5 & 3.8 & 0.9 & 1.1 & 0.5 & 0.6 & 1.2 & 1.3 \\
\hline methanol & 2.8 & 3.0 & 2.9 & 3.2 & 0.4 & 0.2 & 0.1 & 0.2 \\
\hline 2-butanol & 2.6 & 2.4 & 0.6 & 0.8 & 0.6 & 0.9 & 0.1 & 0.3 \\
\hline 1-propanol & 3.9 & 3.8 & 1.7 & 2.0 & 0.2 & 0.3 & 0.2 & 0.3 \\
\hline isobutanol & 5.6 & 5.8 & 1.8 & 2.1 & 0.4 & 0.3 & 0.1 & 0.2 \\
\hline 1-butanol & 0.4 & 0.7 & 0.3 & 0.5 & 0.4 & 0.3 & 0.1 & 0.2 \\
\hline 2-methyl-1-butanol & 3.9 & 4.2 & 0.4 & 0.7 & 0.3 & 0.5 & 0.3 & 0.3 \\
\hline 3-methyl-1-butanol & 4.8 & 5.0 & 0.7 & 0.9 & 0.6 & 0.8 & 0.3 & 0.2 \\
\hline
\end{tabular}

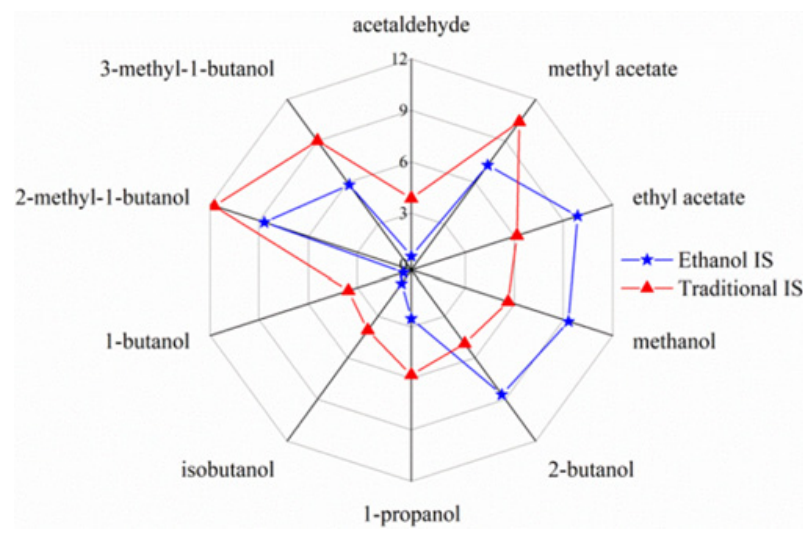

Figure 2. The histogram of biases (\%) comparison for the solution " 0.05 ".

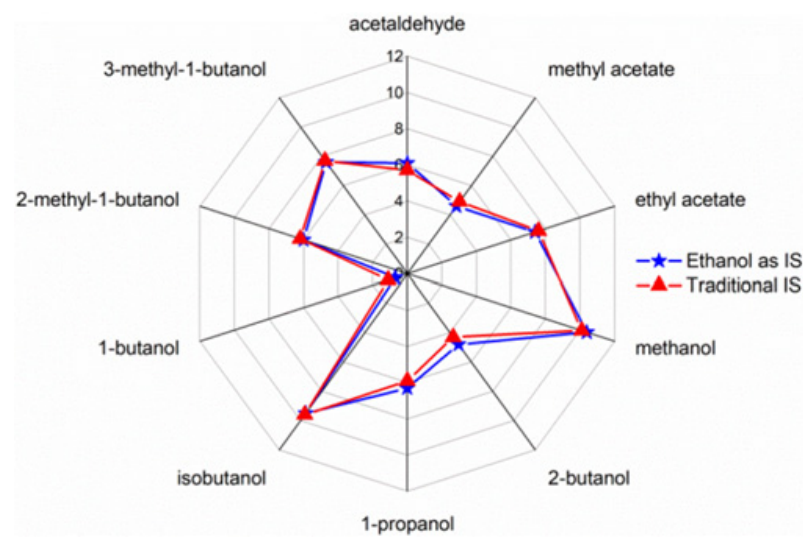

Figure 3. The histogram of LOQ (mg/L AA) comparison.

found in Table 6. According to the presented results, method employing ethanol as an IS compound leads to the precision not worse than that of traditional IS method as corresponding deviations values were generally equal.

Figure 2 depicts the comparison of biases established for the most diluted standard solution " 0.05 ", according to the item 5.14.6 of [4]. It can be seen that although "Ethanol as IS" wasn't better in all cases it still has an adequate accuracy if compared to the currently employed method.

In addition, LOQ values were estimated by the same procedure as it was done in [21] and compared with the used method (Fig. 3). It can be seen that "Ethanol as IS" method got very close LOQ values as those of the traditional IS method.

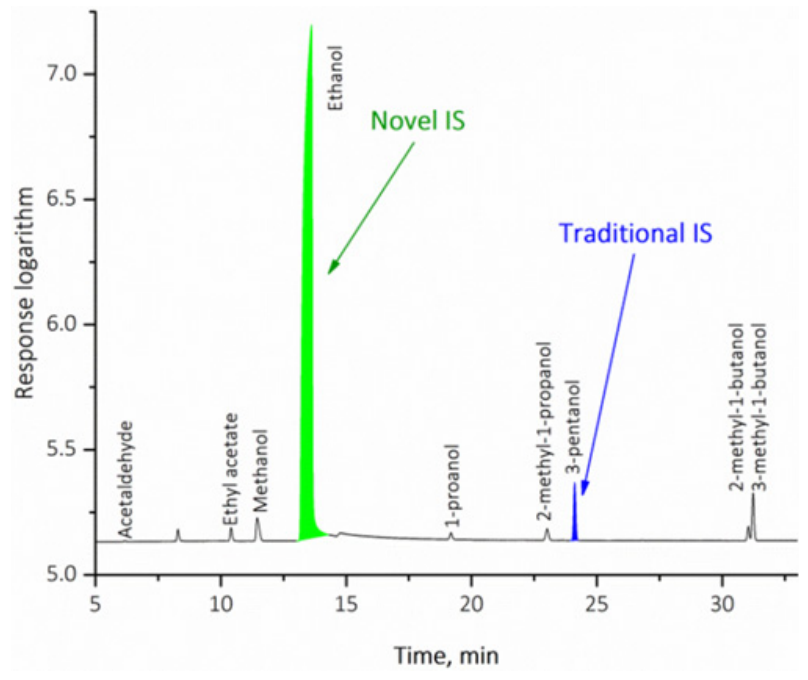

Figure 4. Chromatogram of Wine.

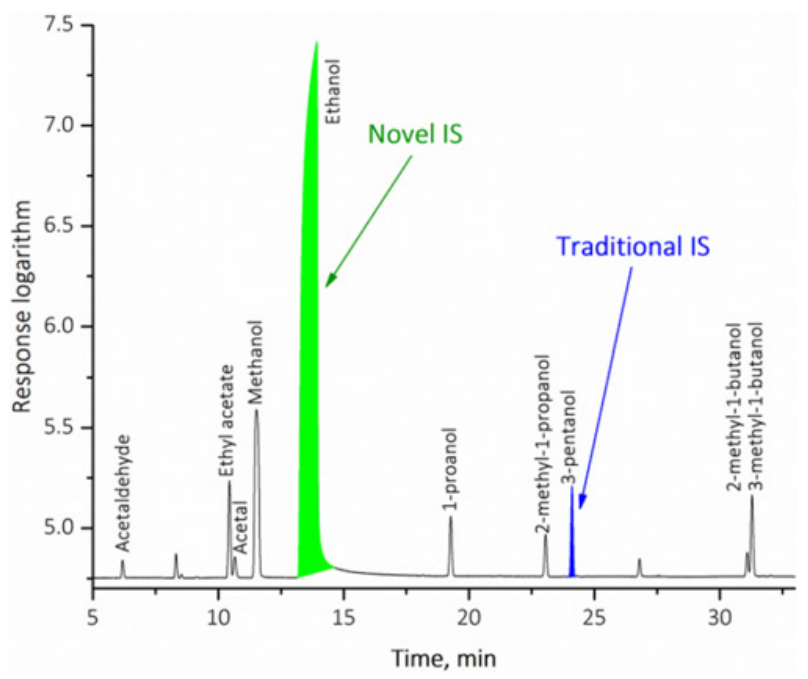

Figure 5. Chromatogram of raki.

To demonstrate if the method is applicable on real samples, the measurements of some alcoholic beverages were evaluated with the suggested method, too. In the Figs. 4 and 5 there are chromatograms of wine and raki, correspondingly, where both used ISs are marked with color.

To evaluate if the difference between the results obtained by two methods is significant Student's t-Test of 
independent samples was used. It was found, that there was no statistical evidence that associated populations means differ significantly for all compounds ( $p=0.05)$.

Eventually we showed that the discussed "Ethanol as Internal Standard" method can be validated according to the previously obtained data only, without any additional measurements or manual procedures. The evaluated metrological properties showed that suggested method can be regarded as accurate and precise.

\subsubsection{Interlaboratory validation}

By analogy with the single laboratory validation algorithm, interlaboratory validation can be done according to the previously obtained data. It is important to note that LGC (formerly the Laboratory of the Government Chemist) prepares certified reference material, such as LGC5100 Whisky-Congeners, for the world market regularly. Before the reference material can be sold interlaboratory experiments are performed. In these experiments $10 \mathrm{~mL}$ aliquots of commercially available whiskey are measured by 16 profile authoritative laboratories. Thus, this procedure can be also used for method validation by contacting LGC with a request of data presentation. It is highly likely that each laboratory prepares standard solutions for calibration, linearity checking and quality control tests. LGC is allowed to request not only measurement results but also accompanying technical and experimental information, including calibration process data. This data would be completely sufficient for the purpose of "Ethanol as Internal Standard" interlaboratory validation.

In addition, the following example of method validation based on interlaboratory tests can also be given. Regularly, twice a year, the Bureau National Interprofessionnel du Cognac carries out interlaboratory comparisons for the quality control of cognac and brandy, in which more than 15 profile laboratories take part.

Let us point out, that such characteristic as trueness (relative biases) can only be evaluated through measurements of gravimetrically prepared set of standard solutions in the framework of the same interlaboratory study. It means that above-mentioned interlaboratory experiments don't provide us with data allowing evaluation of trueness, as no gravimetrically prepared solutions were used. Nevertheless, interlaboratory validation [9] of the traditional IS method which is still used in legislative document [4] didn't include gravimetrically prepared solutions.

\section{Conclusions}

The results of the interlaboratory study of the "Ethanol as Internal Standard" method for the direct quantification of volatile compounds in ethanol-containing products were discussed. It was shown that this method is simpler and cheaper compared to the traditional IS methods and not worse from the precision and accuracy point of view. These benefits originate from the fact that there is no necessity of IS addition into both calibration and test samples. The linearity and precision of the method were not worse than those of the traditional method. Several probable procedures of single laboratory validation and interlaboratory validation of the discussed method were proposed. It was shown that the proposed procedures can be very close to those undertaken earlier [9] during the testing of a method that later became the bedrock of European legislation [4].

It is time to conduct Interlaboratory Study under OIV patronage for the recognition of the new method for official regulatory purposes.

\section{Conflicts of interest}

The authors declare no conflict of interest.

\section{Supplementary materials}

All supplementary materials cited throughout the text of this paper can be found at:

https: //drive.google.com/open?id=1g_TAeiVx wH8zFP5Us6gh6zY-Y13drgQb

Additional information about the method and its application algorithms can be achieved:

- By visiting YouTube channel "Ethanol - IS": https : //www . youtube. com/channel/UCXgL2c__K G3m71W10x0GqtQ

- By direct contacting with the authors via common e-mail: Ethanol. Internal. Standard@gmail . com.

\section{References}

[1] S. Charapitsa, S. Sytova, A. Korban, L. Sobolenko, J. AOAC Int. 102, 669 (2019)

[2] S. Charapitsa, A. Kavalenka, N. Kulevich, N. Makoed, A. Mazanik, S. Sytova, Y. Kotov, J. Agr. F. Chem. 61, 2950 (2013)

[3] S. Charapitsa, S. Sytova, A. Korban, N. Boyarin, I. Shestakovich, R. Čabala, J. Chem. Metr. 12, 59 (2018)

[4] Commission regulation (EC) No 2870/2000

[5] Determination of the principal volatile substances of spirit drinks of viti-vinicultural origin, OIV method (2009)

[6] Alcohol (higher) and ethyl acetate in distilled liquors. Alternative gas chromatographic method. AOAC, Official Method 972.10 (2005)

[7] Chinese National Standard GBT 11858-2008 (2008), https : //www . chinesestandard. net/

[8] Food safety and standards authority of India, FSSAI (2015), https://old.fssai.gov.in/

[9] J. Kelly, S. Chapman, P. Brereton, R. Kiddie, P. Durante, A. Garcia, L. Maignial, M. Williams, A. Bossard, J. AOAC Int. 89, 1375 (1999)

[10] ISO 5725-2: 1994. Accuracy (trueness and precision) of measurement methods and results. Part 2

[11] ISO 5725-4: 1994. Accuracy (trueness and precision) of measurement methods and results. Part 4

[12] M. Thompson, S. Ellison, R. Wood, IUPAC Tech. Rep. 78, 145 (2006)

[13] ISO 17043: 2010. Conformity assessment-General requirements for proficiency testing

[14] Eurachem guide "The fitness for purpose of analytical methods, a laboratory guide to method validation and related topics (2nd ed.)" (2014)

[15] W. Horwitz, J. AOAC Int. 86, 109 (2003) 
[16] Appendix D: Guidelines for Collaborative Study Procedures To Validate Characteristics of a Method of Analysis, AOAC (2002)

[17] Eurolab technical report 1/2007, measurement uncertainty revisited: Alternative approaches to uncertainty evaluation (2007)
[18] Guide quantifying uncertainty in analytical measurement (3rd ed.) Eurachem (2012)

[19] K. Rome, A. Mcintyre, Chrom. Today, 52 (2012)

[20] Commission regulation (EC) No 110/2008

[21] M. Yilmaztekin, T. Cabaroglu, J. AOAC Int. 94, 611 (2011) 